\title{
Neural stem cell-conditioned medium upregulated the PCMT1 expression and inhibited the phosphorylation of MST1 in SH- SY5Y cells induced by $A \boldsymbol{\beta}_{25-35}$
}

\author{
XINWEI WU ${ }^{1}$; GuOYong JIA ${ }^{2, *}$; Hongna YANG ${ }^{3}$; CongCONG SUN ${ }^{2}$; YING LIU ${ }^{2}$; ZeNGYAN DIAO \\ ${ }^{1}$ Department of Geriatrics, Qilu Hospital, Cheeloo College of Medicine, Shandong University, Jinan, China \\ 2 Department of Neurology, Qilu Hospital, Cheeloo College of Medicine, Shandong University, Jinan, China \\ 3 Department of Critical-Care Medicine, Qilu Hospital, Cheeloo College of Medicine, Shandong University, Jinan, China
}

Key words: Neural stem cell conditioned medium, Protein isoaspartate methyltransferase 1, MST1, Amyloid $\beta_{25-35}$, Apoptosis

\begin{abstract}
A progressive neurodegenerative disease, Alzheimer's disease (AD). Studies suggest that highly expressed protein isoaspartate methyltransferase 1 (PCMT1) in brain tissue. In the current study, we explored the effects of neural stem cell-conditioned medium (NSC-CDM) on the PCMT1/MST1 pathway to alleviate A $\beta_{25-35}$-induced damage in SH-SY5Y cells. Our data suggested that $A \beta_{25-35}$ markedly inhibited cell viability. NSC-CDM or Neural stem cell-complete medium (NSC-CPM) had a suppression effect on toxicity when treatment with $\mathrm{A} \beta_{25-35}$, with a greater effect observed with NSC-CDM. A $\beta_{25-35}+$ NSC-CDM group exhibited an increase in PCMT1 expression. sh-PCMT1 markedly decreased cell proliferation and suppressed the protective role of NSC-CDM through the induction of apoptosis and improved p-MST1 expression. Overexpression of PCMT1 reversed the A $\beta_{25-35}$-induced decrease in cell proliferation and apoptosis. In summary, our findings suggest that NSC-CDM corrects the A $\beta_{25-35^{-}}$ induced damage to cells by improving PCMT1 expressions, which in turn reduces phosphorylation of MST1.
\end{abstract}

\section{Introduction}

$\mathrm{AD}$ is a multifactorial disease with multiple risk factors. The pathological mechanisms of $\mathrm{AD}$ include the deposition of amyloid $\beta(A \beta)$, astroglia degeneration, hyperphosphorylation and accumulation of the tau protein, neuronal dystrophy, oxidative stress, mitochondrial dysfunction, biological metal homeostasis, and decreased levels of acetylcholine (ACh) (Butterfield et al., 2001; Reddy, 2011). However, the underline molecular mechanism of $\mathrm{AD}$ is still unclear, and a real cure for the disease has not yet been found.

Previous studies have shown highly expressed protein isoaspartate methyltransferase 1 (PCMT1) in brain tissue (Zhu et al., 2001). Many studies have shown that PCMT1 acts a critical effect in the regulation of longevity, different types of oxidative stress, heat shock response, and apoptosis (Aleksandra et al., 2014; Jost et al., 2002). Recent research has shown that short-term brain tissue injury (such as subarachnoid hemorrhage (SAH) or traumatic brain injury (TBI)) can up-regulate PCMT1 activity. The up-regulation of

*Address correspondence to: Guoyong Jia, jiaguoyong@sdu.edu.cn Received: 07 January 2021; Accepted: 22 March 2021
PCMT1 is related to the decrease of neurodegenerative factor levels and the anti-apoptotic effect of neurons, thereby inhibiting cerebral edema (Liang et al., 2017; Shi et al., 2017a).

MST1 is a pre-apoptotic protein and the key protein of the Hippo pathway. Activation of MST1 can induce the Bax expression, inhibit the $\mathrm{Bcl}-2$ expression, and cause Caspase 3 activation and apoptosis (Qin et al., 2015). Previous studies reported that, in HEK293 cells, PCMT1 and MST1 colocalized, which inhibited cell apoptosis, and after administration of MST1 activator, apoptosis was stronger than without administration, while PCMT1 expression remained unchanged (Biterge et al., 2014).

Neural stem cells (NSCs) are progenitor cells or sources of differentiation of neurons. NSCs have strong self-renewal ability and an important ultra-clean effect in the process of differentiation. A previous study demonstrated that MVs from human NSCs were able to attenuate neuroinflammation and preserve host neuronal morphology in the irradiated brain (Baulch et al., 2016). In addition, transplantation of NSC-CDM into injured mouse brains not only caused expansion of the NSC population in the subventricular zone but also enhanced the formation of new neurons that migrated to the damaged site (Rhee et al., 2016). NSC-CDM was confirmed to have the capacity of 
inducing mesenchymal stem cells into neural stem cell-like cells in vitro (Ma et al., 2011), which further suggested that NSCCDM might enhance endoneurogenesis. Thus, instead of cell transplantation, we use a purified medium containing NSCs paracrine products. Our experiment was to use the protective role of NSC-CDM in cytotoxicity caused by $A \beta_{25-35}$, including the roles of cell viability, cell death, mitochondrial microstructure destruction, and oxidative stress. PCMT1 is stored in mammals and may have a critical effect on the selfhealing process of NSCs. Thus, we hypothesize that NCS-CDM can regulate nerve cell death by regulating the PCMT1/MST1 pathway. These results provide a new target for $\mathrm{AD}$ therapy using a single intervention that has multiple effects.

\section{Materials and Methods}

\section{Bioinformatics analysis}

Download the gene chip data set GSE5281 from the database GEO (http://www.ncbi.nlm.nih.gov/geo). Download the expression matrix from the GEO database for data processing, and then standardize the data obtained, and finally use the Limma package for differential expression analysis. The hippocampus, medial temporal gyrus, and posterior cingulate tissues of $\mathrm{AD}$ patients and normal elderly were analyzed for differential expression, and then the differential genes $(|\log 2 \mathrm{FC}| \geq 1, \operatorname{Padj}<0.05)$ that were up-regulated or down-regulated in the three tissues were screened. 666 differential protein-coding genes were screened. The 666-differential protein-coding genes were screened for GO and KEGG analysis using cluster Profiler. Volcano plots and histograms are completed with ggplot2.

\section{Cell incubation and treatment}

Neural stem cell complete medium (NSC-CPM), including DMEM/F12, human recombinant epidermal growth factor (EGF; $20 \mathrm{ng} / \mathrm{mL}$ ) and basic fibroblast growth factor (bFGF; $20 \mathrm{ng} / \mathrm{mL}$ ), B27 (serum-free medium supplements formulated to provide optimal growth condition for NSC expansion, 1:50), heparin $(5 \mu \mathrm{g} / \mathrm{mL}), 2 \mathrm{mM} \mathrm{L}$-glutamine, and an antibiotic-antimycotic mixture $(1: 100,10,000 \mathrm{U} / \mathrm{mL}$ penicillin, $10,000 \mu \mathrm{g} / \mathrm{mL}$ streptomycin, and $25 \mu \mathrm{g} / \mathrm{mL}$ amphotericin B). Neural stem cell-conditioned medium (NSC-CDM): the cortex region of the E15-18 SD rats was isolated, and the meninges were peeled off on the clean bench. The cortexes were transferred to a $15 \mathrm{~mL}$ conical tube containing $3 \mathrm{~mL}$ HBSS (Hanks' Balanced Salt Solution) for $5 \mathrm{~min}$, then dissociated into small pieces using a $1 \mathrm{~mL}$-pipette tip. $3 \mathrm{~mL}$ of HBSS containing small pieces of cortexes was filtered by $100 \mathrm{~nm}$-filters and centrifuged at $1000 \mathrm{rpm}$ for $10 \mathrm{~min}$ at room temperature to get single cells. After that, the cells were resuspended in a completed culture medium. The SH-SY5Y cells culture and the groups were divided as follows: control group; $\mathrm{A} \beta_{25-35}$ group; $\mathrm{A} \beta_{25-35}+\mathrm{NSC}-\mathrm{CPM}$ group; $\mathrm{A} \beta_{25-35}+$ NSC-CDM group according to our previous study (Jia et al., 2020). Isolating and culturing NSCs and NSC-CDM according to a previous study (Yang et al., 2018).

\section{CCK-8 analysis}

A CCK8 assay kit (Beyotime Biotechnology Co., Ltd., China) was used to evaluate cell viability. SH-SY5Y cells $\left(1 \times 10^{5}\right.$ cells/well) were seeded in 24-well plates and incubated overnight. Next, CCK8 solution $(10 \mu \mathrm{L})$ was added per well and cultured for $4 \mathrm{~h}$. Read the absorbance $(450 \mathrm{~nm})$ by a microplate reader (BioTek, VT, USA).

\section{Apoptosis analysis}

Using an in-situ cell death detection kit (Roche, Mannheim, Germany), the cells were grown on coverslips and terminal deoxynucleotidyl transferase-mediated dUTP nick end labeled with TUNEL for $15 \mathrm{~min}$ at $25^{\circ} \mathrm{C}$. After TUNEL labeling, $0.344-\mathrm{mm}^{2}$ sections were viewed at $400 \times$ magnification making use of a light microscope (BioTek, VT, USA) to quantify apoptotic cells.

\section{Transfections sh-RNA and plasmid of PCMT1}

shRNA targeting PCMT1 and a PCMT1 overexpression vector were synthesized by GenePharma Co. (Shanghai, China). Used the Lipofectamine 2000 reagent (Invitrogen, Carlsbad, CA, USA) to transfect cells with the vector. The experiment is divided into eight groups: (1) control group; (2) NC group; (3) sh-PCMT1; (4) 10\% FBS-containing NSC-CDM + sh-PCMT1 (sh-PCMT1 + NSC-CDM) group; (5) vector; (6) PCMT1-OE; (7) $\mathrm{A} \beta_{25-35} \quad(40 \mu \mathrm{M})$; and (8) $\mathrm{A} \beta_{25-35}+$ PCMT1-OE. After $6 \mathrm{~h}$ of transfection, add DMEM/F-12 complete medium from the 1,2, 3, 7 and 8 group, and for groups 7 and $840 \mu \mathrm{M} \mathrm{A} \beta_{25-35}$ was also added, NSC-CDM containing 10\% FBS for the four groups.

\section{$q R T-P C R$}

The tissues and cell samples RNAs were extracted by RNA Extraction Kit (Promega, Shanghai, China). TB green qPCR master mix (TAKARA BIO INC, Tokyo, Japan) was used for quantitative fluorescence evaluation following with to the manufacturer's requirements. The fluorescence values were expressed by $2^{-\Delta \Delta \mathrm{Ct}}$ and quantified (Livak and Schmittgen, 2001).

\section{Western blot analysis}

First, centrifuged the cell homogenates for $15 \mathrm{~min}$ at $12,000 \times \mathrm{g}$, and collected the supernatants and stored at $-20^{\circ} \mathrm{C}$. Proteins were separated in the supernatants by $12 \%$ SDSpolyacrylamide gels (PAGE) and transferred onto membranes of PVDF. Nonspecific binding was blocked by Tris-buffered saline (TBS) with $3 \%$ BSA (TBS and $0.1 \%$ Tween 20) under gentle shaking at $25^{\circ} \mathrm{C}$ for $1 \mathrm{~h}$. Simultaneously incubated the membranes with the primary antibodies: anti-cleaved caspase- 3 and 9 (1:1000, Applygen, Beijing, China), anti-Bcl-2 and Bax (1:500, Applygen), anti-PCMT1 (1:500, Abcam, USA), total MST1 (1:1000, CST), and p-MST1 (1:1000, Abcam) and anti- $\beta$-actin (1:1000, Abcam) at $4^{\circ} \mathrm{C}$ overnight. Proteins were visualized by a LAS-4000 mini system (Fujifilm, Japan). Protein intensity was quantified using Quantity One software.

\section{Statistical analysis}

Data were analyzed by GraphPad 8.0 and presented as mean \pm standard deviation (SD). Variance (ANOVA) was used to analyze differences among the different groups, PCMT1 expression in three brain regions between patients with $\mathrm{AD}$ and normal elderly were analyzed using the Wilcoxon test. $P<0.05$ was regarded as obvious. 


\section{Results}

The differentially expressed genes of $A D$ patients

Differences in expression were analyzed using the limma editing package in three brain regions (hippocampus, medial temporal gyrus, and posterior cingulate) between patients with $\mathrm{AD}$ and normal elderly. Volcano map of difference analysis results showed that the PCMT1 gene is downregulated in all three brain regions (Fig. 1A). In addition, compared with normal elderly, in $\mathrm{AD}$ patients, the expression of PCMT1 was obviously reduced in the hippocampus, medial temporal gyrus, and posterior cingulate (Fig. 1B, $P<0.001$ ). This suggests that the low function of $\mathrm{PCMT1}$ is a key factor in the poor prognosis of $\mathrm{AD}$.
A

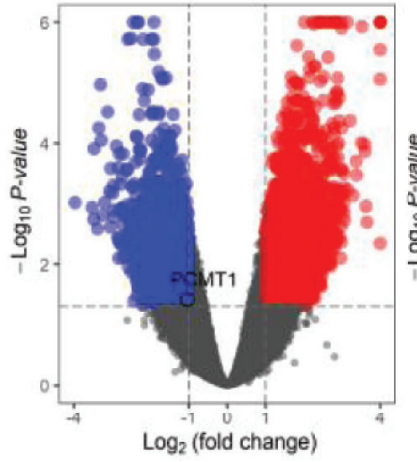

B

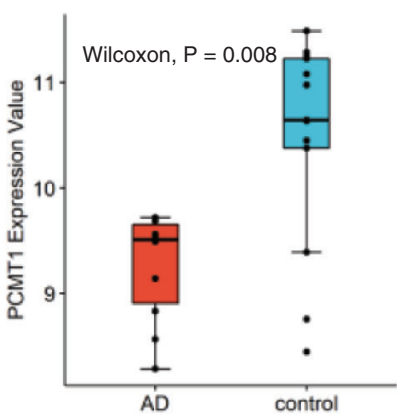

Hippocampus
Medial temporal gyrus

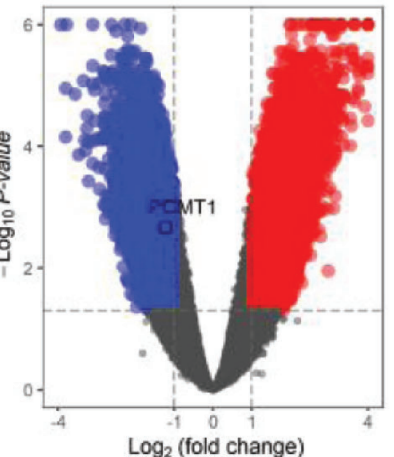

Group 官 AD 官 control

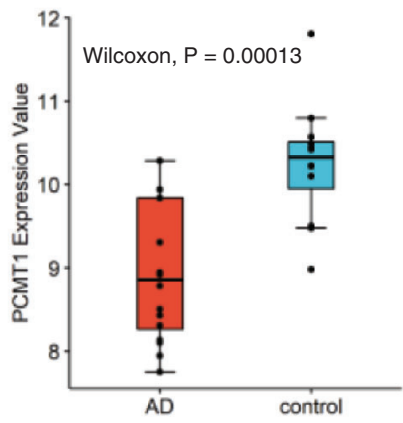

Medial temporal gyrus
Posterior cingulate

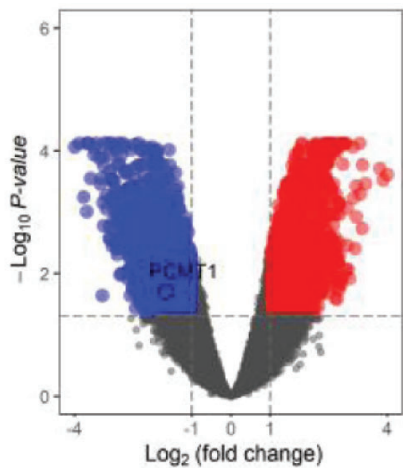

Group 由 AD 官 control

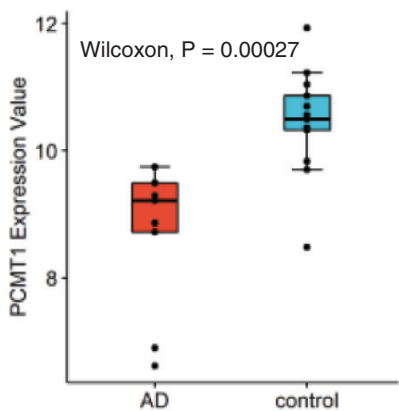

Posterior cingulate
$\mathrm{C}$

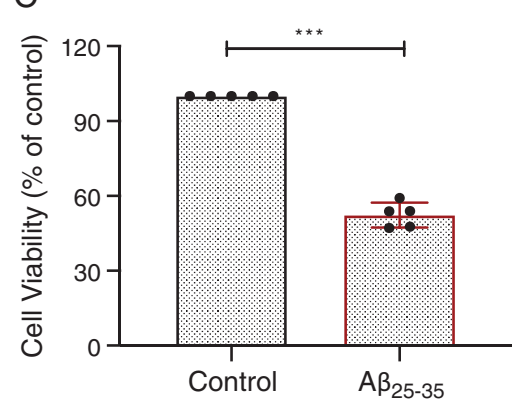

E
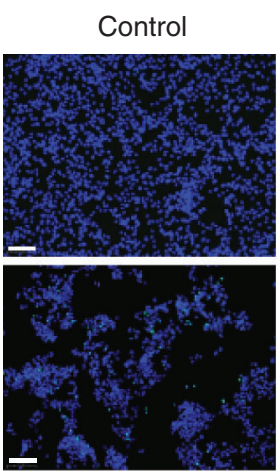

$A \beta_{25-35}+$ NSC-CPM
D
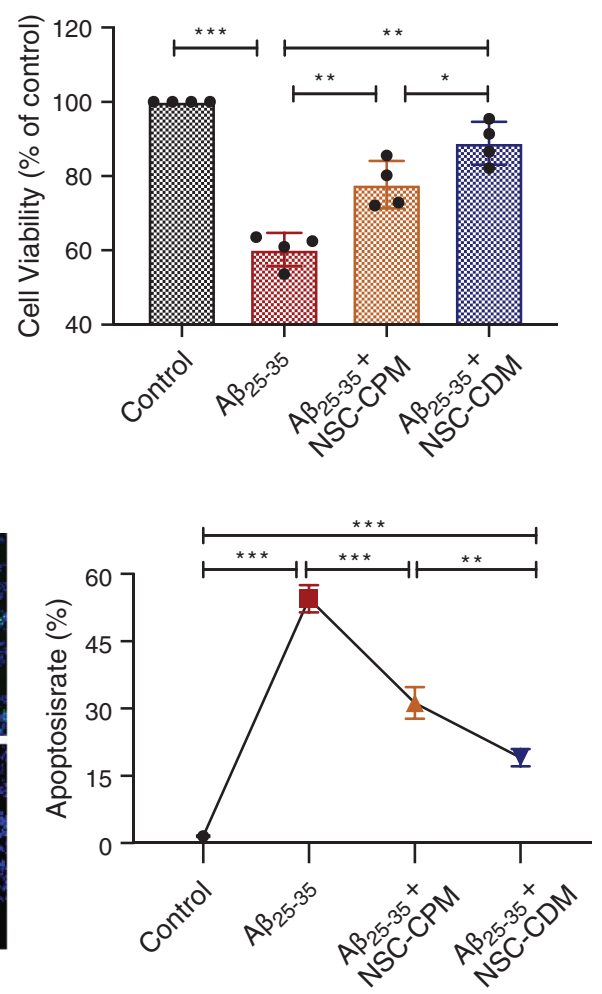

FIGURE 1. Differentially expressed genes of $\mathrm{AD}$ patients and protective role of different media on $A \beta_{25-35}$ damage.

(A) Differential gene distribution map of up-regulated or downregulated genes in three brain regions between patients with $\mathrm{AD}$ and normal elderly. (B) Difference of PCMT1 expression in three brain regions between patients with $\mathrm{AD}$ and normal elderly. (C) CCK-8 detection of cell viability in $\mathrm{SH}$ SY5Y cells treatment of $\mathrm{A} \beta_{25-35}$ (40 $\mu \mathrm{M}, 24 \mathrm{~h}$ ). (D) The cell viability was detected by CCK- 8 assays in four groups for $24 \mathrm{~h}$. (E) TUNEL assay was detected the apoptotic rates of cells in four groups for $24 \mathrm{~h}$. Scale bars: $100 \mu \mathrm{m} .{ }^{\star} P<0.05,{ }^{* *} P<$ $0.01,{ }^{* *} P<0.001$. 
Protective role of different media on $A \beta_{25-35}$-associated damage

Our previous studies confirmed that the modeling condition of the AD cell model was that SH-SY5Y cells treatment of $A \beta_{25-35}(40 \mu \mathrm{M})$ for $24 \mathrm{~h}$ (Jia et al., 2020). Fig. 1C exhibited that compared with the control group, $\mathrm{A} \beta_{25-35}$ obviously inhibited cell viability $(P<0.001)$. NSC-CPM and NSC-CDM exhibited an inhibitory role on the damage $\mathrm{A} \beta_{25-35}$-induced (Fig. 1D) $(P<0.01$ and $P<0.01$, respectively), with the NSC-CDM group demonstrating higher cell viability than the NSC-CPM group $(P<0.05) . \mathrm{A} \beta_{25-35}$-induces apoptosis of SH-SY5Y cells and appears in these cells with the abundance of TUNEL-positive nuclei. The nuclear division of dead cells with concentrated nuclei was also markedly found. The TUNEL-positive nucleus was markedly decreased in the NSC-CDM or NSC-CPM $+\mathrm{A} \beta_{25-35}$-treated cells, with the NSC-CDM group demonstrating a lower number of TUNEL-positive nuclei than the NSC-CPM group (Fig. 1E).
Role of NSC-CPM or NSC-CDM on PCMT1 expression To investigate the role of NSC-CPM or NSC-CDM on PCMT1 levels, the PCMT1 in control, $A \beta_{25-35}(40 \mu \mathrm{M})$, $\mathrm{A} \beta_{25-35}(40 \mu \mathrm{M})+\mathrm{NSC}-\mathrm{CPM}$ and $\mathrm{A} \beta_{25-35}(40 \mu \mathrm{M})+\mathrm{NSC}-$ $\mathrm{CDM}$ group was evaluated by western blotting and $\mathrm{qRT}$ PCR. Compared with the control group, western blotting exhibited a reduction in PCMT1 expressions in the $A \beta_{25-35}$ group $(P<0.01)$. The above effects were ameliorated by NSC-CPM or NSC-CDM, with the NSC-CDM group demonstrating a higher PCMT1 expression than the NSCCPM group (Fig. 2A). Furthermore, mRNA expression of PCMT1 was like the protein expressions of PCMT1 was found (Fig. 2B).

\section{PCMT1 silencing and overexpression}

Compared with the NC group, in the sh-PCMT1 group, the levels of PCMT1 protein and mRNA were markedly reduced (Figs. 2C and 2D). In the sh-PCMT1-2 (48 h) group, the levels of PCMT1 protein and mRNA were minimal. Furthermore, compared with the vector group, the expression
A

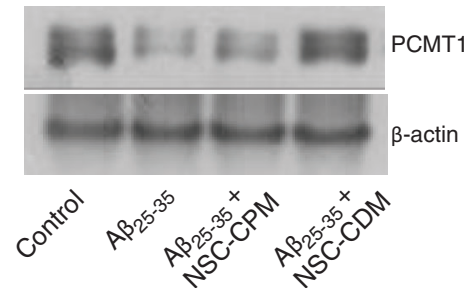

C

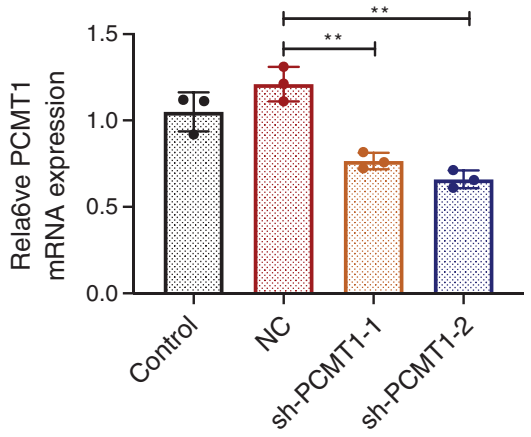

E

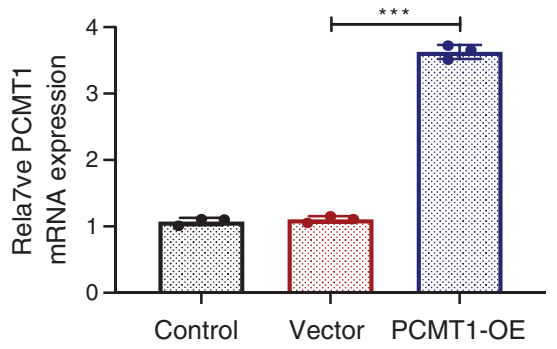

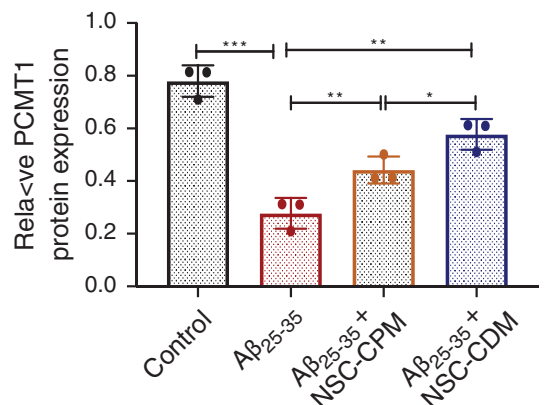

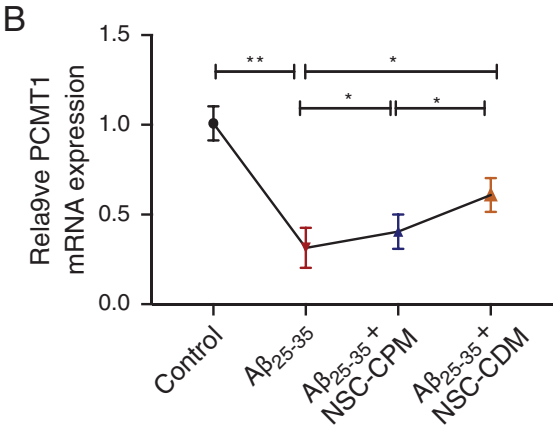

D
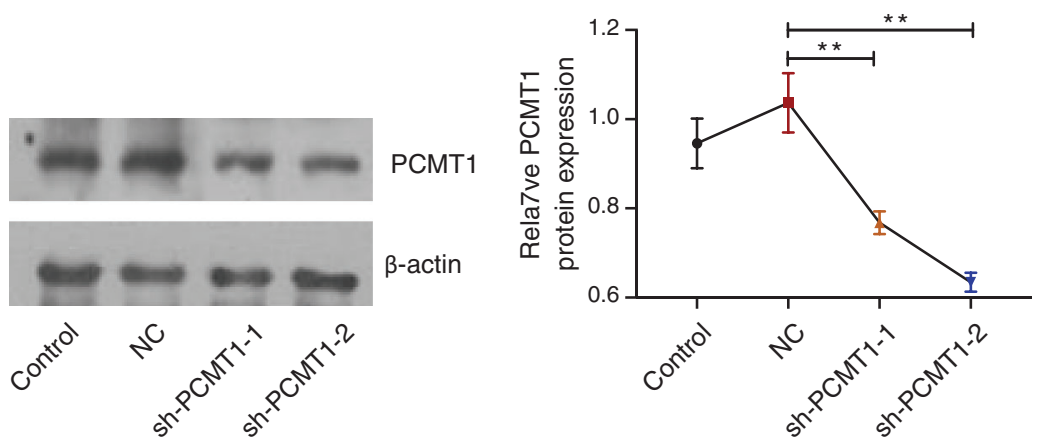

$\mathrm{F}$

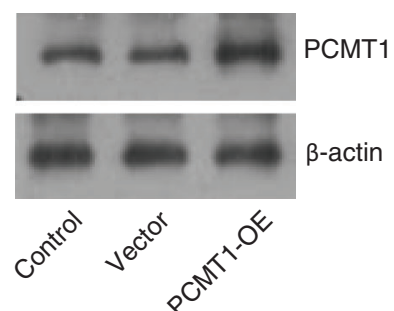

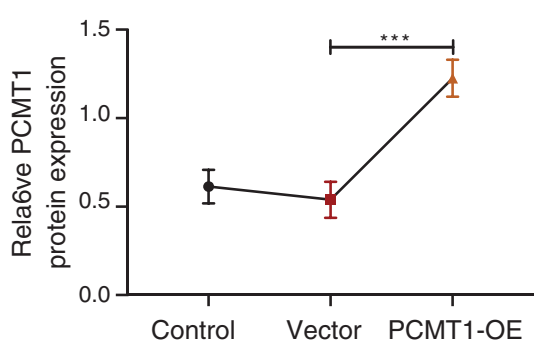

FIGURE 2. Role of NSC-CDM or NSC-CPM on PCMT1 expression.

(A) Western blot was detected the PCMT1 protein expressions in four groups. (B) qRT-PCR detection of the expression of PCMT1 mRNA in four groups. (C) qRT-PCR detection of the expression of PCMT1 mRNA expression in sh-PCMT1 (24 h, $48 \mathrm{~h})$. (D) Western blot detection of the expression of PCMT1 protein expression in sh-PCMT1 (24 h, $48 \mathrm{~h}$ ). (E) qRT-PCR detection of the expression of PCMT1 mRNA expression in overexpression PCMT1 for $48 \mathrm{~h}$. (F) Western blot detection of the expression of PCMT1 protein expression in overexpression PCMT1 for $48 \mathrm{~h} .{ }^{\star} P<0.05,{ }^{* *} P<0.01,{ }^{* *} P<0.001$. 
of PCMT1 mRNA and protein were both obviously improved in the PCMT1-OE group (Figs. 2E and 2F).

Role of PCMT1 on cell proliferation and apoptosis

CCK- 8 results exhibited that cell proliferation was markedly decreased, and the protective role was suppressed after knockdown of PCMT1 compared with the NC group, between the sh-PCMT1 + NSC-CDM group and shPCMT1l group exhibited no statistical difference (Fig. 3A) $(P<0.01$ and $P>0.05$, respectively). Compared with the vector group, in the PCMT1-OE group, greater cell proliferation was found $(P<0.05)$, and the inhibition role of $\mathrm{A} \beta_{25-35}(40 \mu \mathrm{M})$ on cell proliferation was reversed by PCMT1-OE (Fig. 3B) $(P<0.01)$. Furthermore, in the shPCMT1 group, the TUNEL result exhibited that the apoptotic cell numbers were obviously increased compared with the NC group; there was no statistical difference between the sh-PCMT1 + NSC-CDM group and sh-PCMT1 group; PCMT1 overexpressed reversed the effect of $A \beta_{25-35^{-}}$ induced apoptosis (Figs. 3C and 3D).

\section{Effect of PCMT1 on phosphorylation of MST1}

As shown in the Fig. 4A, compared with NC, Bcl2/Bax decreased in sh-PCMT1 group $(P<0.01)$, cleaved caspase $3 /$ caspase 3 was up-regulated $(P<0.05)$; the protection of NSC-CDM was inhibited after PCMT1 silenced (sh-PCMT1 vs. sh-PCMT1 + NSC-CDM, Bcl2/Bax, cleaved caspase3/ caspase 3 had no statistical difference, $P>0.05)$. Sh-PCMT1 treatment significantly enhanced the p-MST1/T-MST1 $(P<0.01)$ as compared to the NC group; p-MST1/T-MST1 was
A
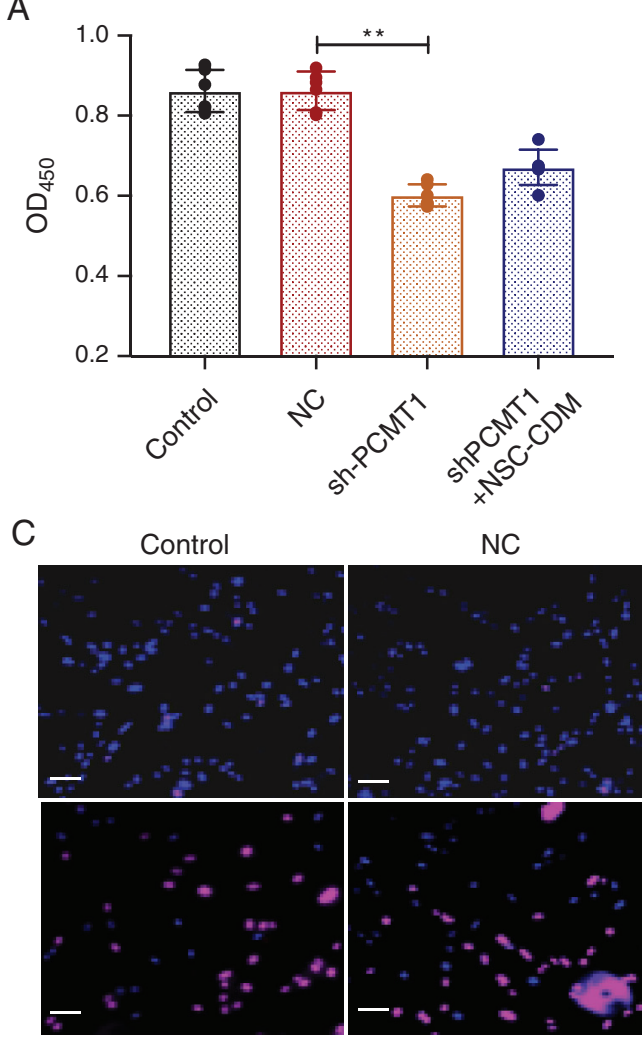

Sh-PCMT1

D

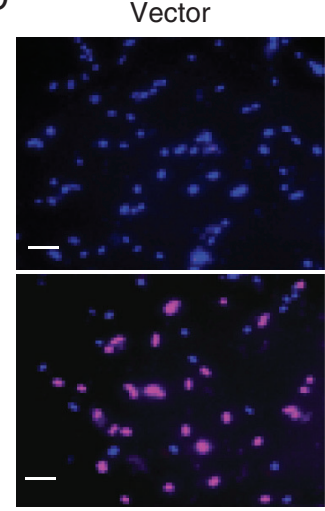

$A \beta_{25-35}$

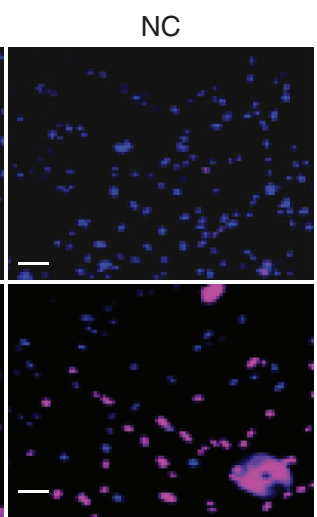

Sh-PCMT1+ NSC-CDM

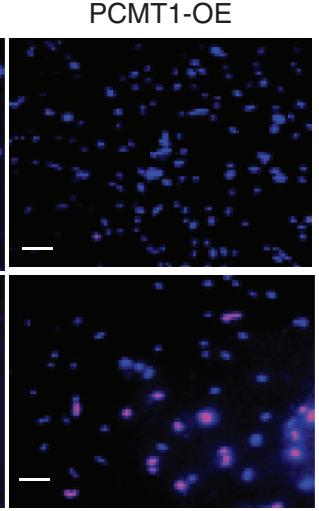

PCMT1-OE+A $\beta_{25-35}$
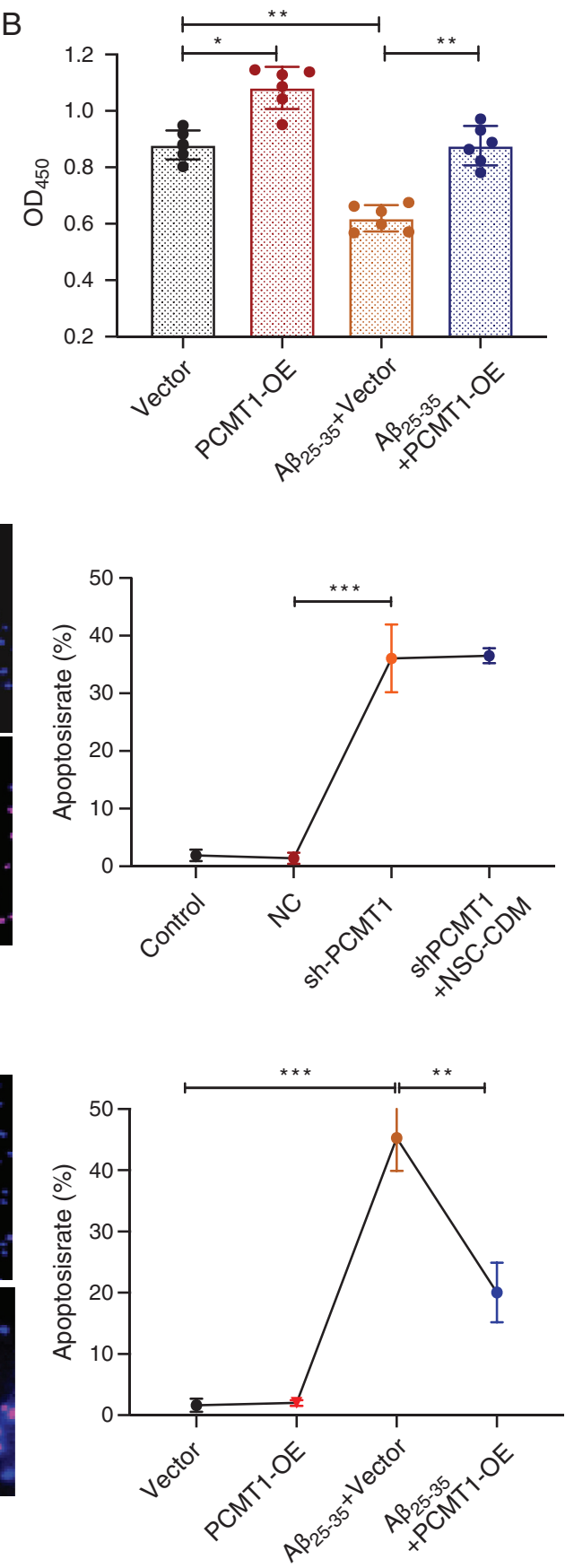

FIGURE 3. PCMT1 increased cell proliferation and inhibited apoptosis. (A) CCK-8 assay was detected the cell viability in control, NC, sh-PCMT1, sh-PCMT1 + NCS-CDM group for $24 \mathrm{~h}$. (B) CCK-8 assay detection of the cell viability in vector, PCMT1OE, $A \beta_{25-35}(40 \mu \mathrm{M})$ and PCMT1$\mathrm{OE}+\mathrm{A} \beta_{25-35}(40 \mu \mathrm{M})$ group for $24 \mathrm{~h}$. (C) TUNEL assay was detected the apoptotic rates of cells in control, NC, sh-PCMT1, sh-PCMT1 + NCSCDM group for $24 \mathrm{~h}$. (D) TUNEL assay was detected the apoptotic rates of cells in vector, PCMT1-OE, $\mathrm{A} \beta_{25-35}(40 \mu \mathrm{M})$ and PCMT1-OE + $\mathrm{A} \beta_{25-35}(40 \mu \mathrm{M})$ group for $24 \mathrm{~h}$. Scale bar: $100 \mu \mathrm{m} .{ }^{\star} P<0.05,{ }^{*} P<$ $0.01,{ }^{* *} P<0.001$ statistical significance. 
A
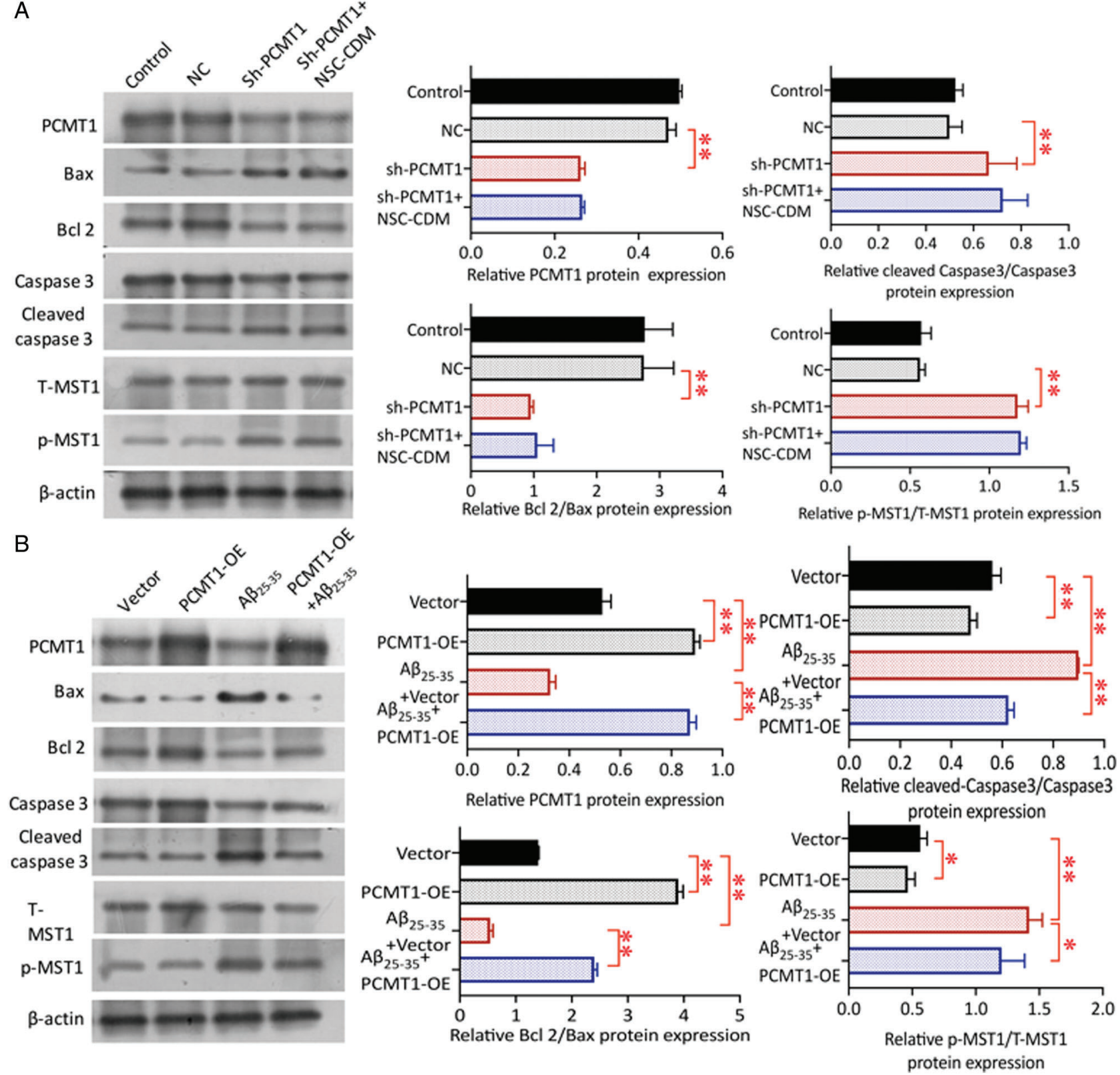

FIGURE 4. Role of PCMT1 on phosphorylation of MST1.

(A) Western blot was detected the PCMT1, Cleaved caspase 3, Caspase 3, Bax, Bcl 2, p-MST1, and T-MST1 protein levels in control, NC, shPCMT1, sh-PCMT1 + NCS-CDM group for $24 \mathrm{~h}$. (B) Western blot was detected the PCMT1, Cleaved caspase 3, Caspase 3, Bax, Bcl 2, p-MST1 and T-MST1 protein levels in vector, PCMT1-OE, A $\beta_{25-35}(40 \mu \mathrm{M})$ and PCMT1-OE $+\mathrm{A} \beta_{25-35}(40 \mu \mathrm{M})$ group for $24 \mathrm{~h} .{ }^{*} P<0.05,{ }^{* *} P<0.01$ statistical significance.

markedly increased; NSC-CDM could not reverse the changes after PCMT1 silenced (sh-PCMT1 vs. sh-PCMT1 + NSC-CDM p-MST1/T-MST1 had no statistical difference, $P>0.05)$. Fig. $4 \mathrm{~B}$ exhibited the level of PCMT1 and $\mathrm{Bcl} 2 / \mathrm{Bax}$ expression in the $\mathrm{A} \beta_{25-35}(40 \mu \mathrm{M})$ +vector group was significantly reduced (all $P<0.01$ ), cleaved caspase3/caspase 3 was increased $(P<0.01)$ compared with the vector group; the expression of above proteins expressions was reversed as compared to the $A \beta_{25-35}$ $(40 \mu \mathrm{M})+$ PCMT1-OE group. The p-MST1/T-MST1 in the $A \beta_{25-35}(40 \mu \mathrm{M})$ +vector group was markedly increased compared with the vector group $(P<0.01)$; after overexpression of PCMT1, the expression of p-MST1/TMST1 was decreased by A $\beta$-induced $(P<0.05)$.

\section{Discussion}

NSCs have the common characteristics of self-renewing stem cells and the characteristics of directed neural differentiation (Hwang et al., 2014; Lewis, 2017). NSC-CDM has been shown to exert antiapoptotic effects in vitro and in vivo (Liang et al., 2014). PCMT1 is highly expressed in brain tissue, including the substantia nigra, blue plaque, and paraventricular nucleus (Zhu et al., 2001). PCMT1 is highly active and pleiotropic, so even small differences in overall PCMT1 activity may have a profound impact on the longterm function of the central nervous system (CNS) (Zhu et al., 2006). Bioinformatics analysis found that PCMT1 activity in the brain tissue of $\mathrm{AD}$ patients was significantly 
decreased (Johnson et al., 1991). Therefore, we speculate that PCMT1, a conservative gene with aging protein repair, has a key effect in the self-renewal process of NSCs, and the paracrine effect of NSCs can up-regulate PCMT1 expression. In this study, we cultured SH-SY5Y cells induced by $A \beta_{25-35}$ with NSC-CPM and NSC-CDM. The results exhibited that the expression of PCMT1 protein and mRNA increased markedly in NSC-CPM and NSC-CDM, and NSC-CDM had a stronger effect than NSC-CPM $(P<0.05)$.

PCMT1 has been associated with apoptosis. Sambri et al. (2011) found that microRNA 15a/16-1 regulates apoptosis in hepatocellular tumor cells through PCMT1, and Liang et al. (2017) showed that CGP3466B plays a neuroprotective role by regulating apoptosis through the PCMT1/Mst1 pathway in rats with traumatic brain injury. MST1 is also involved in tumor occurrence and overgrowth of cells (Perumal et al., 2017), as MST1 deficiency can cause tumor formation that is associated with apoptosis of multiple cell types (Shi et al., 2017b). Overexpression of PCMT1 has been shown to prevent apoptosis induced by Bax in neurons and COS-1 cells. Overexpression of PCMT1 has also been shown to block $\mathrm{H}_{2} \mathrm{O}_{2}$-induced apoptosis through regulation of the methylation of various anti-apoptotic proteins, including Hsp90, Hsp70, and Bcl-xL, in vascular endothelial cells (Bidinosti et al., 2010; Huebscher et al., 1999; Loforese et al., 2017; Maroto and Perez-Polo, 2002). Increased PCMT1 activity is also associated with neuroprotection due to the prevention of neuronal apoptosis and cerebral edema (Liang et al., 2017; Shi et al., 2017a).

The present study demonstrates that $\mathrm{A} \beta_{25-35}$ inhibits cell viability and induces apoptosis in SH-SY5Y cells, which was reversed by treatment with NSC-CDM. In the present study, a decrease in PCMT1 expression levels was observed in the $A \beta_{25-35}$-treated group. However, these effects were ameliorated by treatment with NSC-CDM. CCK- 8 analysis showed that after knockdown of PCMT1 using sh-PCMT1, there was significantly reduced cell viability. Overexpression of PCMT1 resulted in increased cell viability and reversal of the effects of $A \beta_{25-35}$ treatment. In addition, using a TUNEL assay, we found that the number of apoptotic cells was markedly higher in SH-SY5Y cells with sh-PCMT1 treatment, whereas overexpression of PCMT1 reversed the effect of $A \beta_{25-35}$ induced apoptosis. Western blotting showed that sh-PCMT1 and $A \beta_{25-35}$ treatment significantly reduced PCMT1 expression, reduced the ratio of $\mathrm{Bcl} 2 / \mathrm{Bax}$, increased the ratio of cleaved caspase 3/caspase 3 , and increased the ratio of p-MST1/T-MST1. sh-PCMT1 inhibited the protective effects of NSC-CDM, while overexpression of PCMT1 reversed these effects.

\section{Conclusion}

Taken together, the NSC-CDM up-regulates the PCMT1 expression, acts on the classic Hippo apoptosis pathway by inhibiting the activation of MST1, and reduces the apoptosis of $\mathrm{A} \beta_{25-35}$-induced SH-SY5Y cells.

Availability of Data and Materials: The datasets used and/or analyzed during the current study are available from the corresponding author on reasonable request.
Authors' Contribution: WXW, Article writing and revision, data analysis; JGY, study conceptions procurement of samples, paper drafting; YHN, DZY, procurement of samples and results analysis; LY, data collecting and analysis; SCC, study conception and paper drafting. All authors read and approved the final version of the present research manuscript and agreed to be accountable for all aspects of the work.

Ethical Approval: All samples for this study were collected in accordance with the Helsinki Declaration and were approved by the local Ethical Committee.

Funding Statement: The authors received no specific funding for this study.

Conflicts of Interest: The authors declare that they have no conflicts of interest to report regarding the present study.

\section{References}

Aleksandra D, Qin Z, Aswad Dana W, Uversky Vladimir N (2014). Isoaspartyl formation in creatine kinase $\mathrm{B}$ is associated with loss of enzymatic activity; implications for the linkage of isoaspartate accumulation and neurological dysfunction in the PIMT knockout mouse. PLoS One 9: e100622-e100630. DOI 10.1371/journal.pone.0100622.

Baulch JE, Acharya MM, Allen BD, Ru N, Chmielewski NN, Martirosian V, Giedzinski E, Syage A, Park AL, Benke SN, Parihar VK, Limoli CL (2016). Cranial grafting of stem cell-derived microvesicles improves cognition and reduces neuropathology in the irradiated brain. Proceedings of the National Academy of Sciences of the United States of America 113: 4836-4841. DOI 10.1073/pnas.1521668113.

Bidinosti M, Martineau Y, Frank F, Sonenberg N (2010). Repair of isoaspartate formation modulates the interaction of deamidated 4E-BP2 with mTORC1 in brain. Journal of Biological Chemistry 285: 19402-19408. DOI 10.1074/jbc.M110.120774.

Biterge B, Richter F, Mittler G, Schneider R (2014). Methylation of histone $\mathrm{H} 4$ at aspartate 24 by protein L-isoaspartate O-methyltransferase (PCMT1) links histone modifications with protein homeostasis. Scientific Reports 4: 6674-6682. DOI 10.1038/srep06674.

Butterfield DA, Drake J, Pocernich C, Castegna A (2001). Evidence of oxidative damage in Alzheimer's disease brain: Central role for amyloid $\beta$-peptide. Trends in Molecular Medicine 7: 548-554. DOI 10.1016/S1471-4914(01)02173-6.

Huebscher KJ, Lee J, Rovelli J, Ludin B, Matus A, Stauffer D, Fürst P (1999). Protein isoaspartyl methyltransferase protects from Bax-induced apoptosis. Gene 240: 333-341. DOI 10.1016/ S0378-1119(99)00443-6.

Hwang DH, Shin HY, Kwon MJ, Choi JY, Ryu B, Kim BG (2014). Survival of neural stem cell grafts in the lesioned spinal cord is enhanced by a combination of treadmill locomotor training via insulin-like growth factor-1 signaling. Journal of Neuroscience 34: 12788-12800. DOI 10.1523/ JNEUROSCI.5359-13.2014.

Jia G, Diao Z, Liu Y, Sun C, Wang C (2020). Neural stem cellconditioned medium ameliorates $A \beta_{25-35}$-induced damage in SH-SY5Y cells by protecting mitochondrial function. Bosnian Journal of Basic Medical Sciences 24: 1-7.

Johnson BA, Shirokawa JM, Geddes JW, Choi BH, Kim RC, Aswad DW (1991). Protein L-isoaspartyl methyltransferase in 
postmortem brains of aged humans. Neurobiology of Aging 12: 19-24. DOI 10.1016/0197-4580(91)90034-H.

Jost CR, van der Zee CEEM, In'tZandt HJA, Oerlemans F, Verheij M, Streijger F, Fransen J, Heerschap A, Cools AR, Wieringa B (2002). Creatine kinase B-driven energy transfer in the brain is important for habituation and spatial learning behaviour, mossy fibre field size and determination of seizure susceptibility. European Journal of Neuroscience 15: 1692-1706. DOI 10.1046/j.1460-9568.2002.02001.x.

Lewis S (2017). Neurodegenerative disease: Remodelling neurodegeneration. Nature Reviews Neuroscience 19: 3-10.

Liang F, Shi L, Zheng J, Chen S, Wang Y, Zhang J (2017). Neuroprotective effects of CGP3466B on apoptosis are modulated by protein-L-isoaspartate (D-aspartate) O-methyltransferase/Mst1 pathways after traumatic brain injury in rats. Scientific Reports 7: 9201-9209. DOI 10.1038/ s41598-017-08196-3.

Liang P, Liu J, Xiong J, Liu Q, Zhao J, Liang H, Zhao L, Tang H (2014). Neural stem cell-conditioned medium protects neurons and promotes propriospinal neurons relay neural circuit reconnection after spinal cord injury. Cell Transplantation 23: 45-56. DOI 10.3727/096368914X684989.

Livak KJ, Schmittgen TD (2001). Analysis of relative gene expression data using real-time quantitative PCR and the $2-\Delta \Delta \mathrm{CT}$ method. Methods 25: 402-408. DOI 10.1006/meth.2001.1262.

Loforese G, Malinka T, Keogh A, Baier F, Simillion C, Montani M, Halazonetis TD, Candinas D, Stroka D (2017). Impaired liver regeneration in aged mice can be rescued by silencing Hippo core kinases MST1 and MST2. EMBO Molecular Medicine 9: 46-60. DOI 10.15252/emmm.201506089.

Ma K, Fox L, Shi G, Shen J, Liu Q, Pappas JD, Cheng J, Qu T (2011). Generation of neural stem cell-like cells from bone marrowderived human mesenchymal stem cells. Neurological Research 33: 1083-1093. DOI 10.1179/1743132811Y.0000000053.

Maroto R, Perez-Polo JR (2002). Bcl-2-related protein expression in apoptosis: Oxidative stress versus serum deprivation in PC12 cells. Journal of Neurochemistry 69: 514-523. DOI 10.1046/ j.1471-4159.1997.69020514.x.

Perumal N, Perumal M, Kannan A, Subramani K, Halagowder D, Sivasithamparam N (2017). Morin impedes Yap nuclear translocation and fosters apoptosis through suppression of Wnt $/ \beta$-catenin and NF- $\kappa B$ signaling in Mst1 overexpressed HepG2 cells. Experimental Cell Research 355: 124-141. DOI 10.1016/j.yexcr.2017.03.062.
Qin Z, Dimitrijevic A, Aswad DW (2015). Accelerated protein damage in brains of PIMT+/- mice; A possible model for the variability of cognitive decline in human aging. Neurobiology of Aging 36: 1029-1036. DOI 10.1016/j. neurobiolaging.2014.10.036.

Reddy PH (2011). Abnormal tau, mitochondrial dysfunction, impaired axonal transport of mitochondria, and synaptic deprivation in Alzheimer's disease. Brain Research 1415: 136-148. DOI 10.1016/j.brainres.2011.07.052.

Rhee YH, Yi SH, Kim JY, Chang MY, Jo AY, Kim J, Park CH, Cho JY, Choi YJ, Sun W, Lee SH (2016). Neural stem cells secrete factors facilitating brain regeneration upon constitutive RafErk activation. Scientific Reports 6: 32025. DOI 10.1038/ srep32025.

Sambri I, Capasso R, Pucci P, Perna AF, Ingrosso D (2011). The microRNA $15 \mathrm{a} / 16-1$ cluster down-regulates protein repair isoaspartyl methyltransferase in hepatoma cells. Implications for apoptosis regulation. Journal of Biological Chemistry 286: 43690-43700.

Shi L, Al-Baadani A, Zhou K, Shao A, Xu S, Chen S, Zhang J (2017a). PCMT1 ameliorates neuronal apoptosis by inhibiting the activation of MST1 after subarachnoid hemorrhage in rats. Translational Stroke Research 8: 474-483. DOI 10.1007/ s12975-017-0540-8.

Shi L, Lei J, Xu H, Zheng J, Wang Y, Peng Y, Yu J, Zhang J (2017b). Hydrogen sulfide ameliorates subarachnoid hemorrhageinduced neuronal apoptosis via the ROS-MST1 pathway. Oncotarget 8: 73547-73558. DOI 10.18632/oncotarget.20569.

Yang HN, Wang C, Chen H, Li L, Ma S, Wang H, Fu YR, Qu T (2018). Neural stem cell-conditioned medium ameliorated cerebral ischemia-reperfusion injury in rats. Stem Cells International 2018: 1-7. DOI 10.1155/2018/4659159.

Zhu JX, Doyle HA, Mamula MJ, Aswad DW (2006). Protein repair in the brain, proteomic analysis of endogenous substrates for protein L-isoaspartyl methyltransferase in mouse brain. Journal of Biological Chemistry 281: 33802-33813. DOI 10.1074/jbc.M606958200.

Zhu Y, Qi C, Cao W, Yeldandi AV, Rao MS, Reddy JK (2001). Cloning and characterization of PIMT, a protein with a methyltransferase domain, which interacts with and enhances nuclear receptor coactivator PRIP function. Proceedings of the National Academy of Sciences of the United States of America 98: 10380-10385. DOI 10.1073/ pnas. 181347498 . 